\title{
ESTRUCTURA FUNCIONAL DE LOS CENTROS URBANOS DE LA REGIÓN DE LAS HUASTECAS EN MÉXICO
}

La región de Las Huastecas constituye un área periférica, tanto en la escala de todo el país como respecto a las unidades político-administrativas - estados en cuyos límites está situada. Las investigaciones llevadas a cabo en julio de 2001 abarcaron tan sólo una parte de la región situada en la parte norteña del Estado de Veracruz y en la nororiental del de Hidalgo. La capital del primero de los estados mencionados arriba - Xalapa - está situada a unos $350 \mathrm{kms}$ al sur de la zona de estudio (dada la conformación del terreno y la red de caminos la distancia implica unas 10 horas de viaje en autobús). La Huasteca hidalguense (situada en la Sierra Madre Oriental) constituye también la parte del territorio del estado de Hidalgo que más alejada está de la capital.

Los poblados sometidos al análisis funcional difieren entre si en cuanto a su tamaño, el número de habitantes así como la infraestructura social y económica en la que están equipados. Las investigaciones abarcaron a 22 localidades (veáse fig. 2): 10 entre ellas son sedes municipales: Cerro Azul, Chalma, Chinampa de Gorostiza, El Higo, Huejutla de Reyes, Tepetzintla, Platón Sánchéz, San Felipe Orizatlan, Tamiahua y Tempoal.

Asentamientos, según el número de habitantes, 2001

Tab. 1.

\begin{tabular}{|c|c|}
\hline Número de habitantes & Número de asentamientos \\
\hline $1-999$ & 5 \\
\hline $1000-4999$ & 8 \\
\hline $5000-19999$ & 7 \\
\hline Más de 20000 & 2 \\
\hline TOTAL & 22 \\
\hline
\end{tabular}

El presente texto está basado en los resultados de las investigaciones de campo. Los datos estadísticos provienen del Censo General de la Población de México del año 2000. 


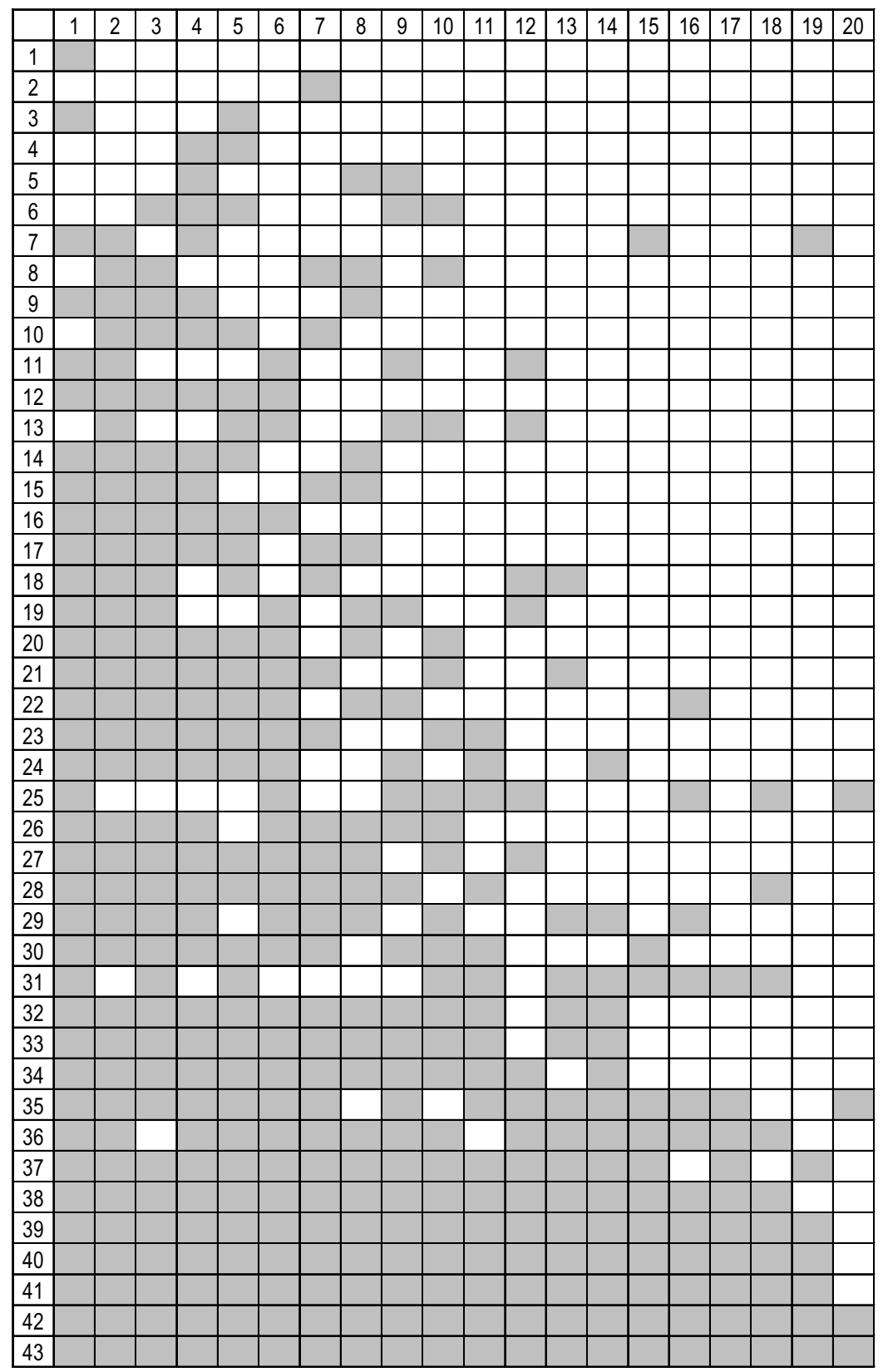

Fig. 1. Distribución de servicios. 
Tomando en cuenta la falta de datos concernientes a la estructura de empleo en cada uno de los asentamientos estudiados se decidió basar el análisis en las informaciones acerca de las formas de actividad económica presentes. Con el fin de facilitar la interpretación de datos provenientes de las encuestas se ha realizado una simple clasificación de asentamientos, según el criterio del número de los establecimientos de servicios, de producción y establecimientos sociales localizados en el lugar. Una clasificación semejante había sido aplicada para el análisis de resultados de las investigaciones realizadas en Las Huastecas en 1985. En la clasificación nueva no se han tomado en cuenta dos centros mejor equipados en la infraestructura y mayores, según el número de habitantes: Huejutla de Reyes y Cerro Azul. La cantidad de establecimientos y el alcance espacial de la mancha urbana no permitieron que se realizaran encuestas — que constituyen puntos de partida para el análisis. Las informaciones provenientes de las entrevistas con las autoridades municipales, asi como las observaciones realizadas permiten, sin embargo, describir el carácter de ambos centros.

En la elaboración de la clasificación se han tomado en cuenta dos componentes: 43 categorías de actividad y 20 localidades donde se han realizado las investigaciones. Las dos localidades de mayor tamaño (Huejutla de Reyes y Cerro Azul) se han excluido de la clasificación, sin embargo su caracteristica se encuentra más abajo. Después de haber ordenado y agrupado los resultados se han definido los cuatro grupos de asentamientos siguientes:

1. Asentamientos con el número de categorías entre 4 y 9.

2. Asentamientos con el número de categorías entre 10 y 19 .

3. Asentamientos con el número de categorías entre 20 y 29.

4. Asentamientos con el número de categorías entre 30 y 36.

El primer grupo está constituido por las localidades más pequeñas y las peor equipadas en elementos infraestructurales: Palma Morelos (cerca de 150 habitantes) y Palmar Prieto (400 habitantes). Palma Morelos es el único asentamiento, entre los visitados, donde la población no dispone de energía eléctrica. En ambas localidades hay escuelas primarias y en Palmar Prieto se encuentran también: un prescolar, un centro médico y una iglesia. El acceso $\leftarrow$

Fig. 1. Distribución de servicios.

Localidades (verticalmente): 1. Tempoal de Sánchez. 2. Tamiahua. 3. San Felipe Orizatlán. 4. El Higo. 5. Platón Sánchez. 6. Tepetzintla. 7. Tehuetlán. 8. Chalma. 9. Coacuilco. 10. Zacamixtle. 11. Chinampa. 12. Tierra Blanca. 13. Juan Felipe. 14. Ixcatlán. 15. La Laja de Colomán. 16. Tampache. 17. El Chote. 18. El Humo. 19. Palmar Prieto. 20. Palma Morelos.

Servicios (horizontalmente): 1. Banco. 2. Cine, teatro. 3. Cafetería. 4. Discoteca. 5. Pulquería. 6. Drenaje. 7. Otra tienda. 8. Otro servicio. 9. Rastro. 10. Sala de billar 11. Venta al por mayor. 12. Electricistas. 13. Frutas y legumbres. 14. Hotel. 15. Restaurante. 16. Supermercado. 17. Preparatoria. 18. Secundaria técnica. 19. Zapatería. 20. Mercado. 21. Refresquerías. 22. Secundaria. 23. Taller mecánico. 24. Taquería. 25. Telebachillerato. 26. Venta de ropa. 27. Peluquería. 28. Acueducto. 29. Carnicería. 30. Sala de baile. 31. Telesecundaria. 32. Farmacia. 33. Ferretería. 34. Panadería. 35. Camión. 36. Prescolar 37. Camino pavimentado. 38. Cantina. 39. Electricidad. 40. Iglesia. 41. Servicios médicos. 42. Tienda miscelánea. 43. Primaria 
a la primera es difícil, ya que no hay camino pavimentado y el camión llega tan solo unas cuantas veces por semana. No hay camiones que lleguen al Palmar Prieto. En cada una de ambas localidades hay varias tiendas misceláneas con productos alimenticios básicos y refrescos. El tipo de construcción de casas es carácteristico también de ambos casos: predominan edificios bajos de una sola planta hechos de varas de bambú con tejados cubiertos de hoja de palma. En el caso de Palmar Prieto se puede hablar, sin embargo, de una polarización del nivel de vida de los habitantes.

$\mathrm{Al}$ segundo grupo pertenecen: El Humo (1 700 hab.), El Chote (500 hab.), Tampache (cerca de 1000 hab.), La Laja de Colomán (alrededor de 500 hab.), Ixcatlán (cerca de 2500 hab.), Juan Felipe (cerca de 3000 hab.) y Tierra Blanca (cerca de 2200 hab.). A semejanza de los asentamientos pertenecientes al primer grupo se trata de poblados agrícolas, sin embargo éstos tienen más habitantes y disponen de la infraestructura mejor desarrollada. Todos tienen electricidad, disponen de escuelas primarias y tiendas con productos básicos, y en la mayoría hay prescolares. En cada una de las localidades existe por lo menos una iglesia. Es importante también el acceso a servicios médicos en el lugar. En Ixcatlán apenás empieza la construcción del camino pavimentado - como la aldea está situada en la sierra, es difícil el acceso. También en Tampache falta camino, sin embargo a todas las localidades, con la excepción de El Humo llegan camiones. Aparte de los dervicios básicos, los habitantes de las localidades mencionadas tienen acceso a escuelas del nivel más alto que el de primaria (secundaria básica, telesecundaria i telebachillerato). También aparecen puestos de comercio o de servicios especializados (panadería, carnicería o taquería).

Al tercer grupo fueron calificadas las localidades siguientes: Chinampa, Zacamixtle, Coacuilco, Chalma, Tehuetlan y Tepetzintla. Todas, aparte de Zacamixtle que cuenta con más de 1200 habitantes, son ciudades pequeñas que agrupan desde 4700 hasta 8000 habitantes. Tres entre ellas son sedes municipales. Todas están equipadas en elementos básicos de la infraestructura social, cuentan con múltiples establecimientos de servicios y de producción artesanal así como con numerosas tiendas especializadas. En Tepetzintla, Chalma y en Zacamixtle por lo menos una vez por semana hay mercados. Los habitantes de la región indicaban a éstos como la fuente principal de abasto no sólo en los productos alimenticios básicos sino también en ropa, calzado, artículos del hogar y otros, lo que pueda influir en que el número de tiendas que ofrezcan dichos productos sea menor. En los mercados hay también puestos de servicios, tales como p.ej. la peluquería. El carácter urbano de las localidades pertenecientes al tercer grupo implica también la ampliación de la infraestructura educativa que abarca también a varios tipos de escuelas del segundo nivel (secundaria básica, telesecundaria, preparatoria). Tanto en las localidades del tercer grupo como en las de los dos primeros casi no existen actividades culturales organizadas, que se limitan a festividades religiosas. La mayoría de localidades de este grupo disponen de una sala de baile. 
En el cuarto grupo se encuentran cinco ciudades más grandes de la región. Todas son centros municipales, a saber: Tempoal de Sánchez (más de 12000 hab.), Tamiahua (más de 5000 hab.), San Felipe Oriztatlán (más de 6000 hab.), El Higo (8 000 hab.) y Platón Sánchez (10 000 hab.). La cifra correspondiente al número de habitantes de Tamiahua suscita dudas. Según la información obtenida de las autoridades municipales, la ciudad la habitan 15000 personas. Las observaciones confirman más bien el valor más alto del dato. Lo indica también el carácter específico de Tamiahua, un pueblo pesquero situados en la orilla de una laguna del Golfo de México, que cumple con múltiples funciones. Su existencia está estrechamnte vinculada a la pesca y la explotación de mariscos. Hay plantas de transformación de mariscos, existe una red de transporte acuático - tanto de mercancía como de pasajeros. Los turistas quienen van de paseo por la aguna o van a la playa del golfo son clientes importantes. Tamiahua es la sede de autoridades municipales, es también un centro de servicios y de comercio - con el mercado regional cada domingo. Es también uno de los pocos centros turísticos en la región.

En los demás centros también podemos observar el desarrollo de infraestructura económica. Disponen de una amplia gama de instituciones educativas, establecimientos comerciales (incluyendo a los que tienen carácter de supermercados), centros de venta a mayoreo; ofrecen tambíen múltiples servicios, entre ellos los más modernos (p.ej. cafés internet). Se trata de localidades conocidas en la región como centros comerciales con los importantes tianguis que atraen al número considerable de comerciantes y de clientes. Es notoria la casi ausencia de establecimientos industriales más grandes. El ingenio en la ciudad de El Higo constituye una excepción. Se nota también el gran número de taquerías, refesquerías o establecimientos gastronómicos de otro tipo. Hay acceso a servicios médicos. Tanto en las localidades de este grupo como en las de los grupos anteriores casi no existen instituciones culturales (cines, teatros, museos) fuera de salas de baile o salones de billar. Los habitantes suelen encontrarse en los zócalos, plazas o calles llenas de gente hasta muy tarde en la noche.

Huejutla (con 34000 hab.) y Cerro Azul (con 21500 hab.) constituyen dos centros urbanos más grandes de la zona del estudio. Ambos tienen carácter de centros regionales, aunque es importante subrayar que en Las Huastecas, fuera de la zona del estudio se encuentran también otros centros urbanos de importancia tales como Tuxpam, Poza Rica o Naranjos, mencionados a menudo por los encuestados como lugares donde la población de la región encuentra trabajo. Huejutla y Cerro Azul destacan entre los centros estudiados no sólo por su tamaño, el número de habitantes, sino también por la infraestructura más rica y el nivel de vida más elevado. Allí se encuentran hospitales, sucursales de bancos, hoteles (en Huejutla son 23), aquí tienen lugar los tianguis más grandes. El desarrollo de cada uno de los centros enumerados a lo largo de los últimos 16 años ha tomado un curso diferente. Mientras que en el caso de Huejutla podemos hablar de la transformación de un pueblo 
pequeño en uno de los centros regionales (con el crecimiento intenso de la población de unos 12 hasta 34 mil durante los 15 años), Cerro Azul, dado el agotamiento de los yacimientos petrolíferos - el factor principal del desarrollo de su economía - a mediadios de los años 80-ta, perdió su importancia en la escala de la región. Junto con el cierre de las instalaciones del PEMEX, la empresa estatal encargada de la explotación y transformación del petróleo la ciudad empezó a despoblarse (el decrecimiento del número de habitantes de 50000 en 1985, a 21500 en 2001), dejando de cumplir el papel del centro educativo más grande - dentro de la zona de estudio, que fue demostrado durante las investigaciones realizadas en Las Huastecas en 1985. Sin embargo, Cerro Azul sigue siendo un centro administrativo con el sector de servicios desarrollado y con múltiples establecimientos comerciales de varios tipos (negocios de lujo, tiendas especializadas, supermercados).

La comparación preliminar de los resultados de investigaciones realizadas en 2001 y las de hace 16 años, basada en los comentarios de las personas encuestadas indica cambios percibidos como positivos en varias aspectos de la vida local. Primero, ha crecido de una manera signifiativa el número de establecimientos educativos. En todas las localidades visitadas se encuentran escuelas primarias, en la mayoría hay prescolares. En total, en la región de estudio funcionan más de 20 escuelas de nivel más elevado que el básico de varios tipos (secundaria, telesecundaria, telebachillerato, secundaria tecnica, preparatoria). La falta de maestros quienes radiquen en el lugar constituye un problema - la mayoría viaja diariamente de los centros urbanos más grandes. Se nota claramente la tendencia de constituir un estandart educativo nuevo - con acceso no solo a la escuela primaria sino también a la educación del segundo nivel en cada localidad. Segundo, ha mejorado la calidad de caminos - a la mayoria de localidades llegan caminos pavimentados. Ha crecido tambien la cantidad de transportistas quienes ofrecen a los habitantes dela región sus servicios. Se trata de unas microempresas de una sola persona, de alcance reducido y el nivel de servicios moderado que responden a la demanda concreta de las sociedades locales. El cambio siguiente consiste en el crecimiento del número de establecimientos comerciales — puestos de venta pequeños. En cada una de las localidades visitadas hay por lo menos dos tiendas miscelaneas que venden productos alimenticios básicos. Su cantidad va creciedo según el tamaño de localidad, p.ej. en Chinampa había 60 establecimientos de ese tipo. Los habitantes entrevistados mencionaban también cierta mejora en cuanto a la accesibilidad y calidad de los servicios médicos, sin embargo siguen siendo raros los casos de médicos quienes vivan en las aldeas. La falta del alcantarrillado en la mayoría de las localidades visitadas constituye un problema grave (tan sólo 6 entre las 22 centros visitados disponen de canalización). La mayoría de los centros disponen tan sólo del desagüe pluvial. Es preocupante también el hecho de que ni las autridades municipales, ni los habitantes mismos mencionen otros problemas ecológicos (p.ej. basura alrededor de las casas), que se notaban claramente durante las visitas en los poblados. En muchas de las localidades más pequeñas falta agua 


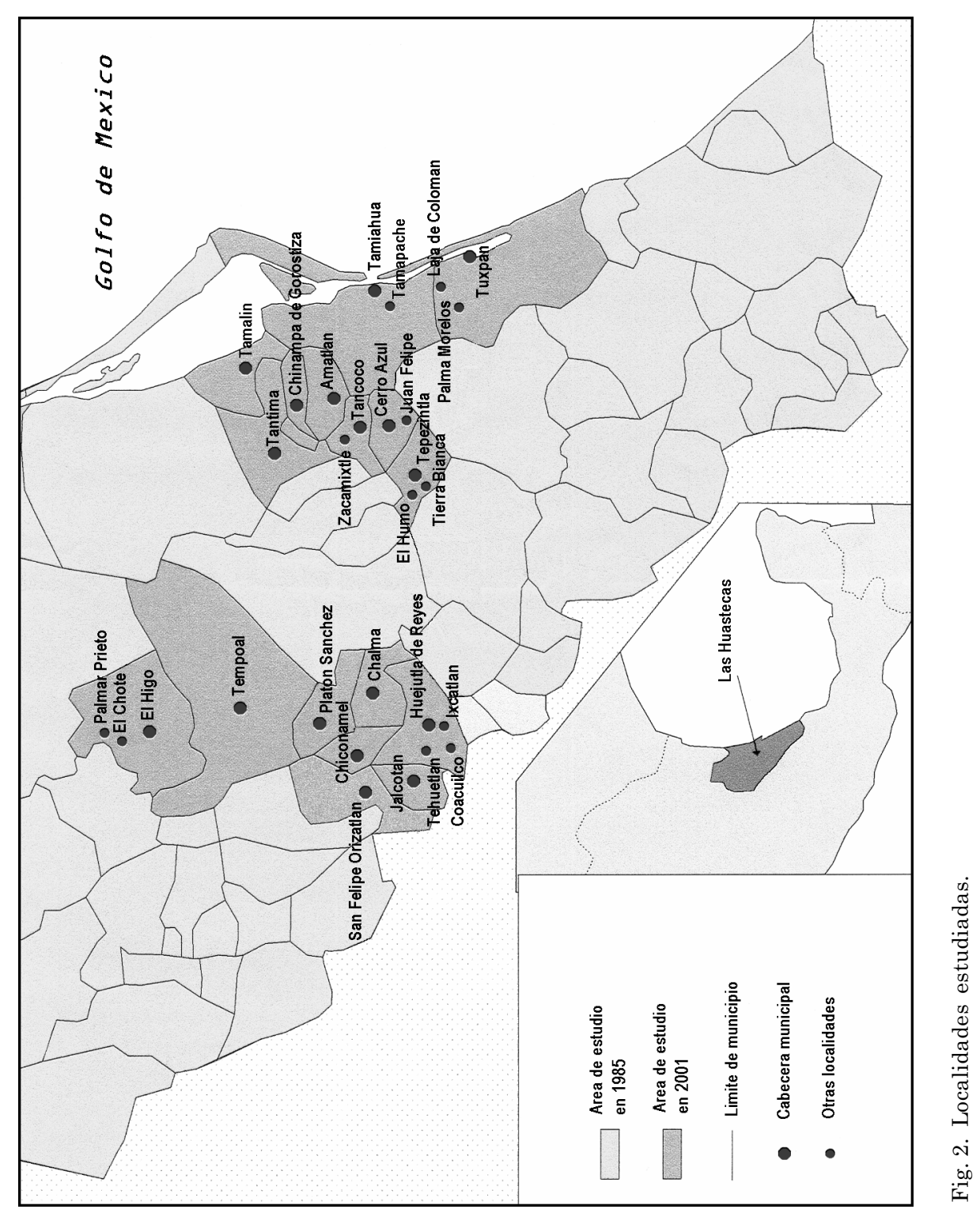


potable. La traen los distribuidores de los centros más grandes, o en los casos extremos es indispensable acarrearla de la fuente.

\section{BIBLIOGRAFÍA}

XII Censo General de Población y Vivienda 2000, INEGI, México.

Czerny M., 1987, Osadnictwo w Las Huastecas [Red de asentamientos humanos en Las Huastecas], [en:] Dembicz A., Dutkiewicz P. (eds), Lokalne problemy wsi regionu Las Huastecas w Meksyku [Problemas locales del campo de la región de Las Huastecas], WGiSR UW, Instytut Gospodarki Przestrzennej, Warszawa.

Skoczek M., 1987, Cechy infrastruktury społecznej na poziomie lokalnym [Rasgos de la infraestructura social], [en:] Dembicz A., Dutkiewicz P. (eds), Lokalne problemy wsi regionu Las Huastecas w Meksyku [Problemas locales del campo de la región de Las Huastecas], WGiSR UW, Instytut Gospodarki Przestrzennej, Warszawa. 